\title{
Diffusion-weighted imaging of the spine using radial k-space trajectories
}

Olaf Dietrich $^{1}$, Amy Herlihy ${ }^{2}$, Wayne R. Dannels ${ }^{3}$, Jochen Fiebach ${ }^{1}$, Sabine Heiland ${ }^{1}$, Joseph V. Hajnal ${ }^{2}$, Klaus Sartor ${ }^{1}$

${ }^{1}$ Department of Neuroradiology, University of Heidelberg Medical School, Heidelberg, Germany

${ }^{2}$ Robert Steiner MRI Unit, Hammersmith Hospital, Imperial College of Science, Technology and Medicine, London, UK

${ }^{3}$ Marconi Medical Systems, MR Division, Cleveland, Ohio, USA

\section{ELECTRONIC PREPRINT VERSION:}

Not for commercial sale or for any systematic external distribution by a third party

Final version: Magn Reson Mater Phy (MGAMA) 2001; 12(1): 23-31.

<URL:http://dx.doi.org/10.1016/S1352-8661(00)00132-0>

\section{Abstract}

Introduction: Diffusion-weighted MR imaging (DWI) of the spine requires robust imaging methods, that are insensitive to susceptibility effects caused by the transition from bone to soft tissue and motion artifacts due to breathing, swallowing, and cardiac motion. The purpose of this study was to develop a robust imaging method suitable for DWI of the spine.

Methods \& Subjects: A radial $k$-space spin echo sequence has been implemented, which is selfnavigating because each acquisition line passes through the origin of $k$-space. Influence of cardiac motion and associated flow of cerebrospinal fluid is minimized by cardiac gating with a finger photoplethysmograph. The sequence has been tested on a 1.5 T system. Diffusion-weighted images of 6 normal volunteers were acquired in the sagittal plane with $4 b$ values between 50 and $500 \mathrm{~s} / \mathrm{mm}^{2}$. Because of the symmetries of the cord, diffusion measurements in the head-foot (HF) or left-right (LR) directions were sufficient to measure the dominant effects of anisotropy.

Results: The apparent diffusion coefficients (ADCs) measured respectively in the LR and HF directions were $(0.699 \pm 0.050) \cdot 10^{-3} \mathrm{~mm}^{2} / \mathrm{s}$ and $(1.805 \pm 0.086) \cdot 10^{-3} \mathrm{~mm}^{2} / \mathrm{s}$ in the spinal cord,
$(1.588 \pm 0.082) \cdot 10^{-3} \mathrm{~mm}^{2} / \mathrm{s}$ and $(1.528 \pm 0.052)$. $10^{-3} \mathrm{~mm}^{2} / \mathrm{s}$ in the intervertebral disks, and $(0.346 \pm$ $0.047) \cdot 10^{-3} \mathrm{~mm}^{2} / \mathrm{s}$ and $(0.306 \pm 0.035) \cdot 10^{-3} \mathrm{~mm}^{2} / \mathrm{s}$ in the vertebrae of the cervicothoracic spine.

Conclusion: Diffusion-weighted spin echo sequences with radial trajectories in $k$-space provide a means of achieving robust, high quality diffusion-weighted imaging and measuring ADCs in the spine. The application of the diffusion-weighting gradients in different direction allows diffusion anisotropy to be measured.

\section{Key words:}

Diffusion-weighted MRI, Human spine, Radial $k$-space trajectory

\section{Correspondence to:}

\section{Olaf Dietrich}

Department of Neuroradiology

University of Heidelberg Medical School

Im Neuenheimer Feld 400

D-69120 Heidelberg, Germany

Telephone: +49-6221-567569

Fax: +49-6221-564673

E-mail: od@dtrx.net 


\section{Introduction}

Diffusion-weighted MR imaging (DWI) has been shown to be valuable in diagnosis and monitoring of stroke due to its high sensitivity for cerebral ischemia within minutes after the onset of symptoms [1-5]. Other clinical applications include characterization of tumors $[6,7]$ or multiple sclerosis lesions $[8-10]$ and white-matter fiber-tract imaging by determining diffusion anisotropy [11-14].

However, neuroradiological application of DWI still focuses on the brain, whereas diffusion imaging of the spinal cord suffers from several technical difficulties. The main problem in DWI in vivo and especially in DWI of the spine are artifacts due to physiological motion. DWI is sensitive to stochastic motion of water molecules in the micrometer range and thus image data can be severely degraded even by sub-millimeter motion of the subject during the acquisition. The main sources of motion in DWI of the spine are breathing, swallowing, cardiac motion, and flow artifacts from cerebrospinal fluid (CSF) and from major vessels close to the spine. Some of the most important approaches to reduce motion artifacts are single-shot imaging $[15,16]$, navigator echo techniques [17-21], and special acquisition schemes with improved inherent robustness against motion like line scan imaging $[22,23]$ or backprojection reconstruction of radial acquisitions [24-26] or combinations of these methods [27,28]. Currently the most common method for brain diffusion imaging studies is single-shot echo planar imaging (EPI) because of low motion sensitivity due to its short acquisition time $[29,30]$.

A considerable problem in DWI of the spine is susceptibility effects caused by the transition from bone to soft tissue or from tissue to air spaces. Such transitions are much more prominent around the spine than in the brain. Single-shot EPI is particularly vulnerable to susceptibility-induced field inhomogeneity.

Hence, DWI of the spine requires especially robust imaging methods. Robustness against susceptibility variations can be provided by spin echo acquisition schemes and motion tolerance can be achieved using radial (or polar) trajectories in Fourier space [31]. This means that $k$-space is covered by a radial pattern of straight trajectories with equal angular spacing and with each passing through its center, $k=0$. Thus, all readout lines are symmetric to the origin of Fourier space and equivalent with respect to the reconstruction. This is not the case in conventional Cartesian readout schemes, where a single corrupted data line can degrade the complete image, especially if it is positioned close to the center of $k$-space.
There are two commonly used methods to reconstruct an image from radially acquired $k$-space data, that can be summarized as backprojection and regridding. The former starts with a one-dimensional Fourier transform of each readout line resulting in parallel projection views of the imaged objects along a rotating axis. These projections can be transformed into the final image using standard algorithms for filtered backprojection (FBP). To improve the robustness of this method, the phase information of the (complex) projections can be discarded before applying the FBP algorithm [31].

The alternative reconstruction method resamples the $k$-space data into a Cartesian grid; this can be done using a variety of interpolation techniques [32, 33]. The final image is reconstructed by a standard twodimensional Fourier transform. Motion influences can be suppressed by appropriate processing of the data before and during regridding.

The purpose of this study was to develop a robust imaging method for measuring apparent diffusion coefficients (ADCs) in the human spine.

\section{Methods \& Subjects}

We have implemented a self-navigating radial spin echo sequence [34]; the sequence scheme is shown in Fig. 1. Both in-plane imaging gradients ( $x$ and $y$ direction) work as frequency-encoding gradients. They are scaled by $\sin \theta_{i}$ and $\cos \theta_{i}$, respectively, thus resulting in radial acquisition lines through the origin of $k$-space. These lines are (incrementally) rotated to a set of angles $\theta_{i}$ in the range $0^{\circ} \leq \theta_{i}<180^{\circ}$ measured from the axis $k_{y}$.

The diffusion gradients in $x$ and $y$ direction can be operated either in a "fixed" or in a "rotating" mode. The former is the conventional configuration usually applied in DWI with diffusion gradients remaining at constant direction with respect to the examined patient. The measured diffusion coefficient is the component of the diffusion tensor that corresponds to the selected diffusion gradient direction.

Alternatively, the diffusion gradients can be scaled by $\cos \theta_{i}$ and $-\sin \theta_{i}$, so that the resultant is always orthogonal to the imaging gradients. This configuration results in a diffusion weighting averaged over all in-plane directions. In some tissues the diffusion properties depend strongly on the direction; e.g. the ADCs are typically increased when measured parallel to tissue fiber direction. With rotating diffusion gradients, it may be advisable to choose the imaging plane orthogonal to 


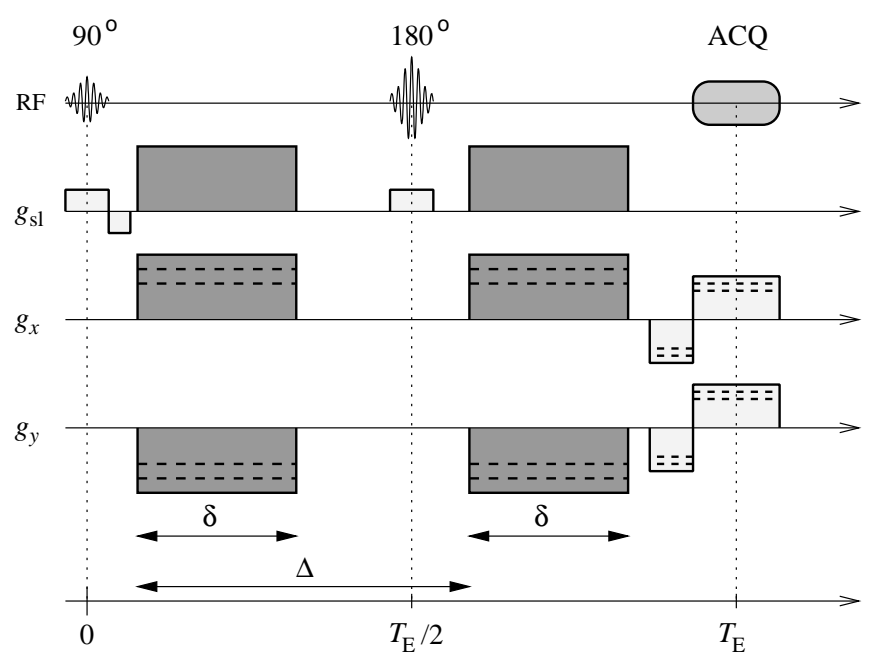

Figure 1: Sequence scheme of "radial" spin echo sequence. Readout gradients in $x$ and $y$ direction are scaled with $\sin \theta$ and $\cos \theta$, respectively. All possible diffusion gradients are shown (dark colored), however typically they are switched on separately. They can be rotating perpendicular to the readout gradients as schematically displayed in this figure or can be static as in conventional DWI.

the fiber direction, so that the diffusion properties remain approximately uniform in the different directions of the rotating diffusion gradients.

To correct for subject motion, several steps of post-processing are applied to the acquired Fourier space data as shown in Fig. 2. Rigid body motion of the imaged object consists of translation and rotation. A translation $\Delta r$ during the diffusion-sensitizing period between the diffusion gradient lobes $g$ results in global phase shift by an angle $\phi_{\mathrm{t}}$, that is proportional to the scalar product of $g$ and $\Delta r: \phi_{\mathrm{t}} \propto g \cdot \Delta r$. Additionally, a (small) rotation described by the axial vector $\Delta \psi$ causes a linear phase shift by $\phi_{\mathrm{r}}(x) \propto(g \times \Delta \psi) \cdot x$ depending on the position $x$ within the imaged object. The constant phase $\phi_{\mathrm{t}}$ remains unchanged after transformation into $k$-space, whereas the linear phase $\phi_{\mathrm{r}}(x)$ is transformed to a coordinate translation by $\Delta k \propto g \times \Delta \psi$.

Each acquired echo has a $k$-space trajectory $z k_{\theta}$ described by a parameter $-1 \leq z \leq 1$ and a vector $k_{\theta}$ in $k$-space rotated by the angle $\theta$ from the axis $k_{y}$. Using rotating diffusion gradients as described above, such that $g$ is orthogonal to $k_{\theta}$ and in the imaging plane, the coordinate translation $\Delta k$ caused by rotational motion is parallel to $k_{\theta}$. This translation of the echo along the readout direction can be corrected by determining the position of the maximum intensity peak of each data line and subsequently shifting this peak to the center $k=0$ (Fig. 2b). The remaining constant phase factor $\exp i \phi_{\mathrm{t}}$ is determined by comparing the complex phase of the echoes at the center of $k$ space $k=0$, that is covered by all trajectories. Since the phase of all echoes should be identical at $k=0$, we multiply all data lines with the appropriate phase factor (Fig. 2c).

With fixed diffusion gradients, this correction scheme does not correct for all possible types of rotational motion. Those $k$-space data regions where the readout trajectories are close to orthogonal to the resulting diffusion gradient are corrected efficiently, as described above. However, when the the readout trajectory becomes parallel to the diffusion gradient direction or generally with diffusion gradients perpendicular to the imaging plane, some but not all components of bulk rotation can be corrected. Especially rotational motion that causes a shift $\Delta k$ with components perpendicular to $k_{\theta}$ cannot be corrected.

In addition to rigid body motion, more complex types of motion can occur such as regional or pulsatile motion. If these components of motion become too strong, they will typically lead to non-linear phase shifts in the imaged object causing broader peaks with reduced maximum intensity in $k$-space. So we can apply a threshold operation to reject echoes degraded by regional motion. For example, we reject any line if its peak intensity is lower than $40 \%$ of the maximum intensity of the 4 neighboring lines (Fig. 2d). The value of $40 \%$ was chosen in earlier experiments, by testing several threshold levels on the same acquired raw data and selecting the threshold which minimized artifact, as measured by signal levels in regions in air, outside the body.

Finally, the acquired data is regridded and then Fourier transformed. Regridding is based on the algorithm described in [33] involving a window function that is convolved with the $k$-space data during regridding. The kernel size used was $4 \times 4$. No additional correction such as synthesizing missing data is needed for the rejected lines, since the regridding algorithm itself inherently compensates for non-uniform sampling in $k$-space.

The sequence has been tested on a clinical 1.5 T Edge imager (Marconi Medical Systems, Cleveland, Ohio) with PD250 gradients (maximum gradient strength $27 \mathrm{mT} \mathrm{m}^{-1}$, maximum slew rate $72 \mathrm{mT} \mathrm{m}^{-1} \mathrm{~ms}^{-1}$ ). We acquired diffusion-weighted images in the sagittal plane of 6 normal volunteers (age 26 to 35); informed consent was obtained from all subjects. RF signals were received with a phased array coil dedicated to spine examinations. Influence of cardiac motion and associated CSF flow was minimized by cardiac gating with a finger photoplethysmograph (PPG); the acquisition was triggered about $400 \mathrm{~ms}$ after the PPG signal maximum. Additionally, we applied a 


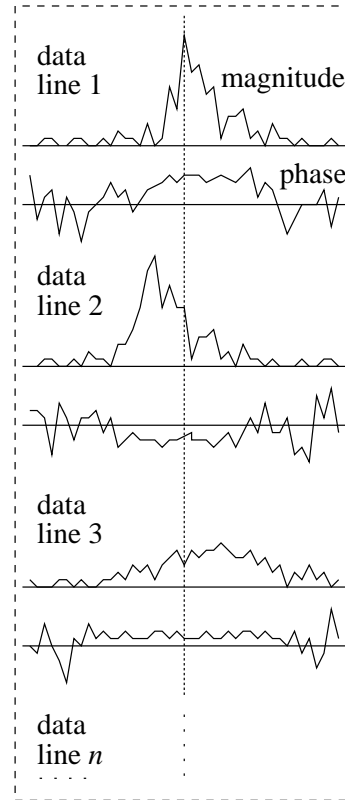

a) original $k$-space data

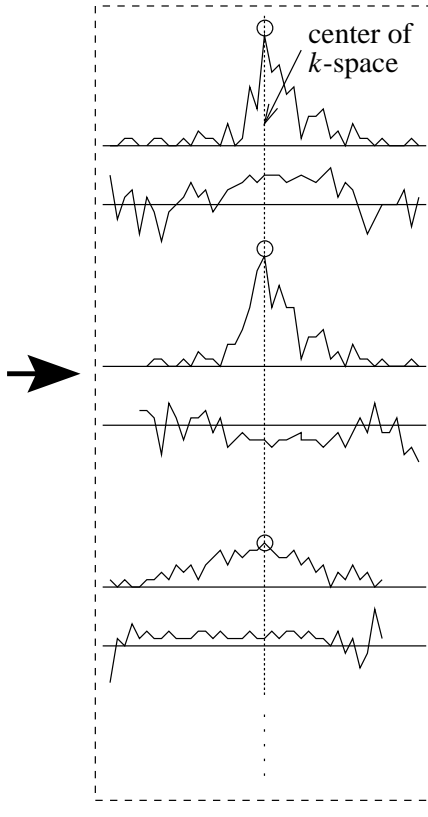

b) shift intensity peaks

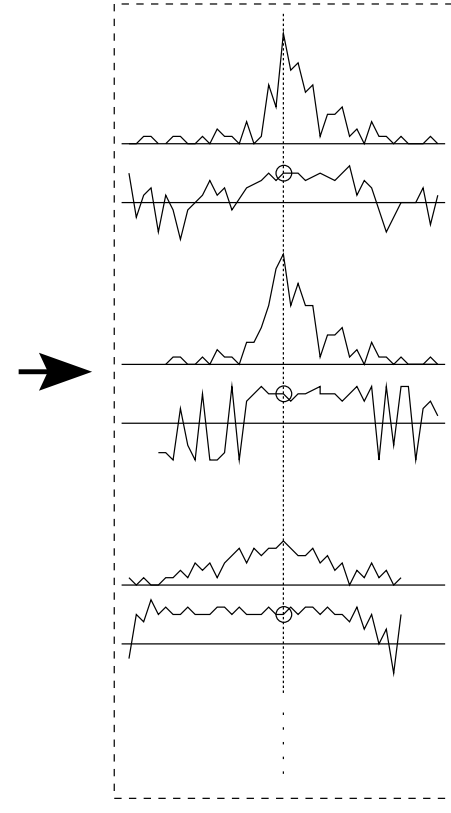

c) apply phase factor

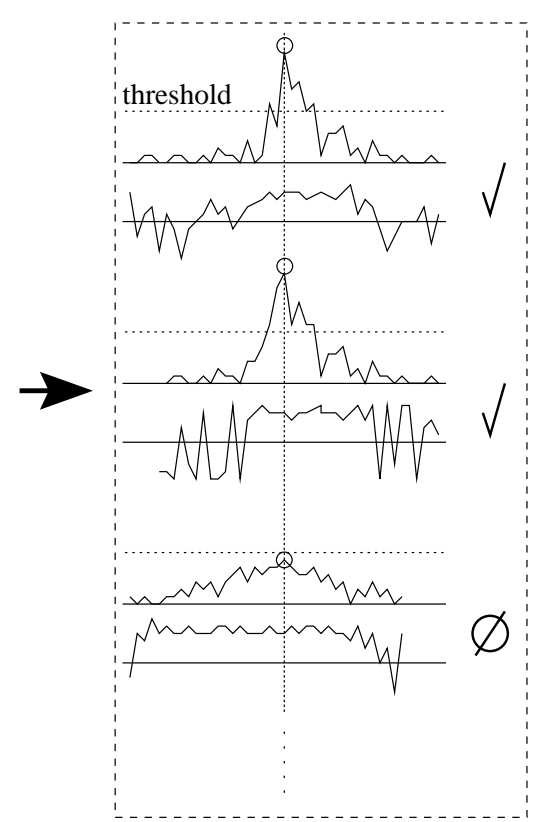

d) reject peaks $<$ threshold

Figure 2: Motion correction scheme. The original data lines (a) are centered at $k=0$ (b), then (c) a phase factor is applied to yield identical complex phases at $k=0$, and finally (d) data lines with peaks lower than $40 \%$ of the maximum intensity of the 4 neighboring peaks are rejected from further processing.

saturation pulse anterior to the spine to suppress image artifacts caused by cardiac motion and blood flow. A second saturation pulse was positioned posterior to mask the intense signal of the subcutaneous fat.

The field of view (FOV) of each acquisition was $20 \mathrm{~cm}$ and was positioned around the transition from cervical to thoracic spine. The resolution in the readout (radial) direction was 128 pixels with an oversampling factor of 2. To avoid streaking artifacts, we acquired $128 \times \pi / 2=201$ echoes, thus obtaining the same sampling density at the perimeter of sampled $k$-space as within each readout. The echo time $\left(T_{\mathrm{E}}\right)$ was $70 \mathrm{~ms}$ and the repeat time $\left(T_{\mathrm{R}}\right)$ was set to one cardiac cycle, thus varying between 800 and $1100 \mathrm{~ms}$; the resulting acquisition time was between $160 \mathrm{~s}$ and $220 \mathrm{~s}$ for each $b$ value. The slice thickness was $4 \mathrm{~mm}$ and a single slice centered in the spinal cord was acquired.

Since we were interested in the main diffusion components of the spinal cord in sagittal slices, we used fixed diffusion gradients in head-foot (HF) and left-right (LR) direction. (A rotating diffusion direction in the sagittal plane would have mixed diffusion coefficient parallel and perpendicular to the spinal cord.) The diffusion gradient duration was $\delta=22.8 \mathrm{~ms}$ and the interval between the onsets of diffusion lobes $\Delta=33.5 \mathrm{~ms}$. The $b$ values quantifying the diffusion weighting were $50,150,250$, and $350 \mathrm{~s} / \mathrm{mm}^{2}$ in $\mathrm{HF}$ direction and 50, 200, 350, and 500 $/ \mathrm{mm}^{2}$ in LR direction. These different ranges were chosen because a higher ADC can be expected in HF direction (paral- lel to the fibers of the spinal cord) resulting in stronger signal decrease at increasing $b$ values. The relatively low maximum $b$ values were used because of the reduced signal-to-noise ratio (SNR) due to the surface coil and the generally higher sensitivity to artifacts in DWI of the spine compared to the brain. ADC maps were calculated from the images on a pixel by pixel basis using the logarithm of the intensity and standard linear regression methods. ADC values were similarly determined by region of interest (ROI) from the diffusion-weighted images. Diffusion anisotropy was described by the anisotropy index, that is defined as $2 \lambda_{1} /\left(\lambda_{2}+\lambda_{3}\right)$ with the main diffusivities, i. e. the eigenvalues $\lambda_{i}$ of the diffusion tensor ordered by magnitude. This is approximately equivalent to $\mathrm{ADC}_{\max } / \mathrm{ADC}_{\min }$ in the case of cylindrical symmetry as in the spinal cord if the ADCs are measured in the approximate directions of the eigenvectors. As discussed below, the curvature of the spine can be neglected without considerably increasing the error of the anisotropy measurements.

In addition to the study of the cervicothoracic spine, we acquired a set of diffusion-weighted images of the whole spine as well to test the robustness of the sequence; the $b$ value for these images was $200 \mathrm{~mm}^{2} / \mathrm{s}$ with diffusion gradients in LR direction. 


\section{Results}

Figure 3 shows examples of images acquired with our radial spin echo DWI sequence $\left(b\right.$ value of $50 \mathrm{~s} / \mathrm{mm}^{2}$ in Fig. $3 \mathrm{a}$ and $b$ value of $350 \mathrm{~s} / \mathrm{mm}^{2}$ in Fig. 3b; diffusion weighting in HF direction). The images have low levels of overt motion artifacts and their resolution is consistent with the effective $128 \times 128$ matrix. Spinal cord, intervertebral disks, and vertebrae can be well distinguished; the signal of CSF around the spinal cord is suppressed by the applied diffusion weighting. The global intensity of the image is decreasing in anterior direction (from right to left) due to the sensitivity profile of the array of surface coils.

With the empirically determined threshold of $40 \%$, the number of rejected lines was below 5 in $85 \%$ of all acquisitions of this study proving the robustness of the acquisition scheme; the maximum number of rejected lines in a single acquisition was 14. A higher number of rejected lines should be expected when imaging a typical patient population because of the usually less 'ideal' cooperation compared to volunteers.

The position of the ROIs used for determining ADCs of the spine of normal volunteers is displayed in Fig. 3c. We examined the diffusion properties of the spinal cord, the disks, and the main part of the vertebral bodies. Since we found a certain variance of the ADC values depending on the exact position of the chosen ROI, we defined multiple ROIs for each image and each type of tissue. All ADCs for a certain tissue from all volunteers were evaluated as one large data set.

Because of a relatively large variance of the determined data we used the median, which is more robust than the arithmetic mean, and the standard deviation of median to characterize our measurements. The results are displayed in Table 1 and in Fig. 4. The ADCs of the spinal cord differ significantly measured in LR and in HF direction indicating a strong anisotropy; the anisotropy index of the spinal cord is $2.58 \pm 0.22$. The ADCs of both the disks and the vertebrae show no significant difference in LR and HF direction, indicating almost isotropic diffusion.

Figure 5 shows ADC maps of the spine of a normal volunteer with diffusion gradients in HF direction (Fig. 5a) and in LR direction (Fig. 5c). The statistical error (obtained by linear regression analysis) of the calculated ADCs is displayed in Fig. 5b and 5d. Background areas and tissue signal suppressed by saturation pulses have been set to zero; the remaining image containing the spinal canal, spinal cord, and the vertebrae has a low level of artifacts.
Images with moderate diffusion weighting covering the whole spine are shown in Fig. 6. As before, these images demonstrate the stability of the sequence and absence of degrading artifacts. The SNR was 8.6 in the cervical, 12.5 in the thoracic, and 5.5 in the lumbar spinal canal.

\section{Discussion}

Diffusion-weighted spin echo sequences with radial trajectories in $k$-space provide a means of achieving robust, high quality diffusion-weighted imaging and measuring ADCs in all areas of the spine. We have not observed a special sensitivity to susceptibility effects as with single-shot EPI, and the SNR is improved compared to techniques like single-shot FSE, HASTE, or line scan imaging because of the shorter echo time and lower bandwidth or larger excited volume, respectively. These advantages compensate for the longer scan time than in single shot techniques; the resulting acquisition time of about 3 minutes for each $b$ value should still be tolerable for most patients. However, volunteers or patients often move slightly during the acquisition of a full set (with multiple $b$ values) of images. Such a shift should be corrected before the calculation of ADC maps to avoid artifacts at the edges or borders of different types of tissue. Partial volume effects are another source of biased results; especially for relatively small structures like the cervicothoracic disks and tissue with changing through-slice profile such as the spinal cord. To minimize the influence of partial volume effects in the spinal cord, we chose a small slice thickness of $4 \mathrm{~mm}$ and used $T_{2}$-weighted coronal localizer scans to position the sagittal slice exactly in the center of the cord.

The choice of a minimum $b$ value of $50 \mathrm{~mm} / \mathrm{s}^{2}$ instead of 0 has the advantage that perfusion effects are suppressed, that lead to non-exponential signal decrease at small $b$ values [35]. In addition, image quality is improved by the suppression of blood and CSF. However, normal diffusion cannot explain the complete signal loss in CSF spaces that can be seen in Fig. 3 at relatively low $b$ values such as $50 \mathrm{~mm} / \mathrm{s}^{2}$. This signal loss is probably caused by additional phase cancellations due to the distribution of flow velocities within a single voxel, resulting in suppressed CSF signal and consequently biased values of the ADC in the CSF (Fig. 5).

The known cylindrical symmetry of the spinal cord has the advantage that the diffusion anisotropy can be estimated with diffusion measurements in only two different directions (parallel and perpendicular 

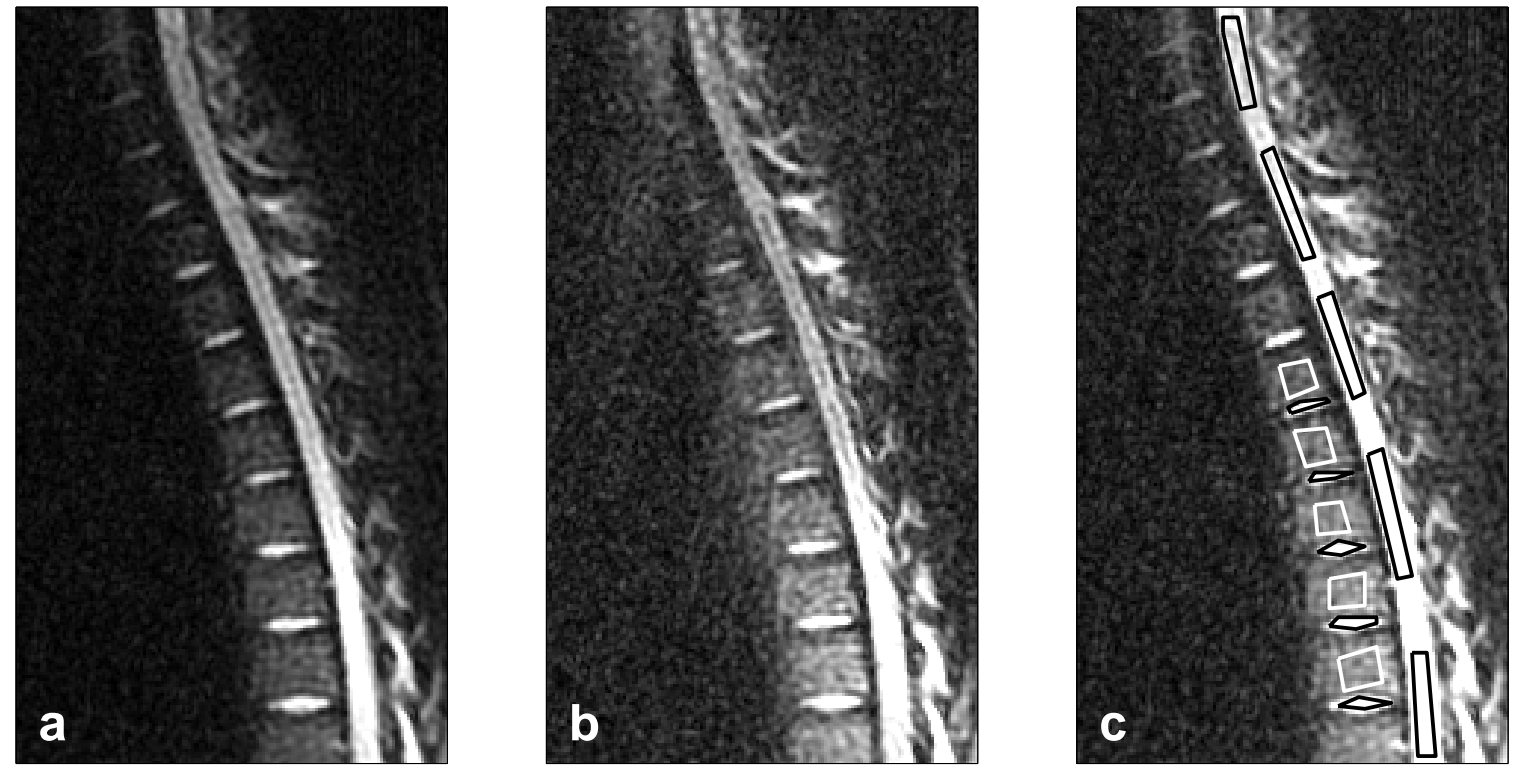

Figure 3: Examples of radially acquired diffusion-weighted images of the spine with diffusion gradients in HF direction and (a) $b$ value $50 \mathrm{~s} / \mathrm{mm}^{2}$ and (b) $b$ value $350 \mathrm{~s} / \mathrm{mm}^{2}$. The images are differently scaled to compensate for signal decrease due to diffusion weighting. Typical positions of ROls for determining ADCs of the spinal cord, disks (both drawn in black), and vertebrae (in white) are shown in (c).

Table 1: ADCs of spinal cord, intervertebral disks, and main part of the vertebral bodies of normal volunteers and the number of ROls used to determine these data. The values are the median and the standard deviation of the median of the measured data. The anisotropy index is given by $\mathrm{ADC}_{\max } / \mathrm{ADC}_{\min }$.

\begin{tabular}{lllll}
\hline Tissue & $\begin{array}{l}\text { Number } \\
\text { of ROIs }\end{array}$ & $\begin{array}{l}\mathrm{ADC} /\left(10^{-3} \mathrm{~mm}^{2} / \mathrm{s}\right) \\
\text { in LR direction }\end{array}$ & $\begin{array}{l}\mathrm{ADC} /\left(10^{-3} \mathrm{~mm}^{2} / \mathrm{s}\right) \\
\text { in HF direction }\end{array}$ & $\begin{array}{l}\text { Anisotropy } \\
\text { index }\end{array}$ \\
\hline Spinal cord & 30 & $0.699 \pm 0.050$ & $1.805 \pm 0.085$ & $2.58 \pm 0.22$ \\
Disks & 30 & $1.588 \pm 0.082$ & $1.528 \pm 0.053$ & $1.04 \pm 0.06$ \\
Vertebrae & 36 & $0.346 \pm 0.047$ & $0.306 \pm 0.035$ & $1.13 \pm 0.20$ \\
\hline
\end{tabular}

to the fiber). This reduces the scan time compared to DWI of the brain where typically the full diffusion tensor with six independent components must be determined. However, this simplified calculation of the anisotropy is not quite correct because the spine is typically curved so that the HF axis is not uniformly parallel to the fibers along the whole length of the spinal cord. Hence, the measured ADCs in HF direction are likely to be slightly too low for most points in the cord. However, this error was neglected in this study; using orthogonal transformations of the diffusion tensor, it can be estimated to be lower than $5 \%$ of the determined ADCs as long as the angle between spine and diffusion gradient direction is below $15^{\circ}$.

We have not applied rotating diffusion gradients in this study because images were acquired only in the sagittal plane with high in-plane diffusion anisotropy. This means, rotating diffusion gradients lead to varying diffusion weighting in different (orthogonal) sections of $k$-space. Thus, the resulting ADC in strongly anisotropic tissue depends not only on its anisotropy but also on its geometric shape and edge orientation because these properties correspond to the different regions in $k$-space, and hence, the obtained data become difficult to interpret. In future studies and patient examinations, it might be desirable to acquire axial slices as well, e. g. using a single sagittal diffusion-weighted image to localize the region affected by a lesion. It may be desirable to use rotating diffusion gradients in axial slices for the improved motion correction as described above.

Preliminary experiments with diffusion sensitization in AP direction demonstrated higher levels of artifact than in the other directions. This may be due to anterior tissue motion caused by the heart and the lungs, that was not completely masked by the applied saturation pulse. Rotating diffusion gradients in the sagittal plane, irrespective of the difficulties mentioned above, suffered from similar effects. 

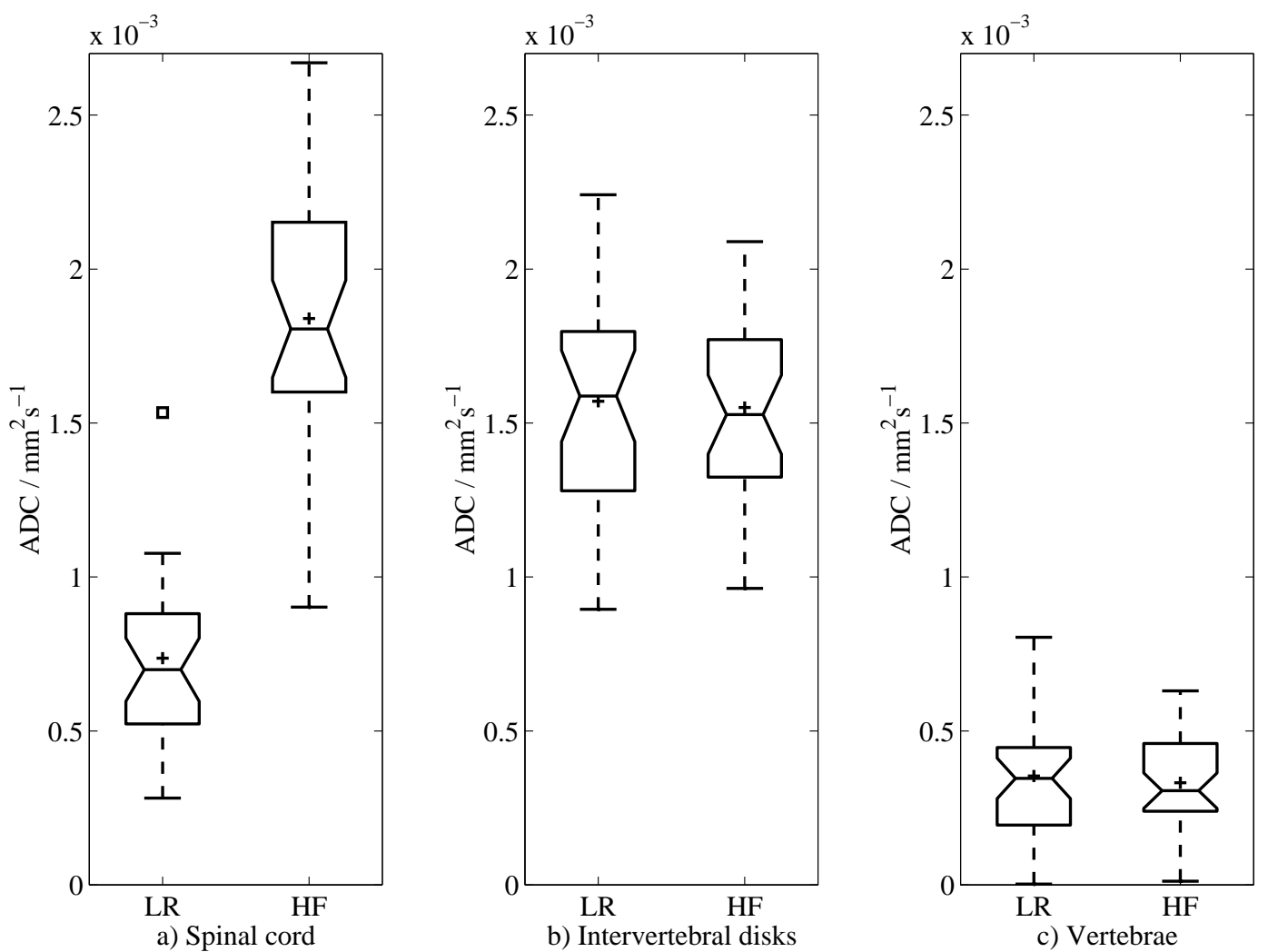

c) Vertebrae

Figure 4: Box plots of ADC values of the spine. The box plots display median (central line) and arithmetic mean (plus sign), the $95 \%$ confidence intervals (notches), the first and third quartile (boxes), and the range of the data sets without outliers (whiskers). Outliers are more than $150 \%$ of the interquartile range removed from the median and are displayed as squares.
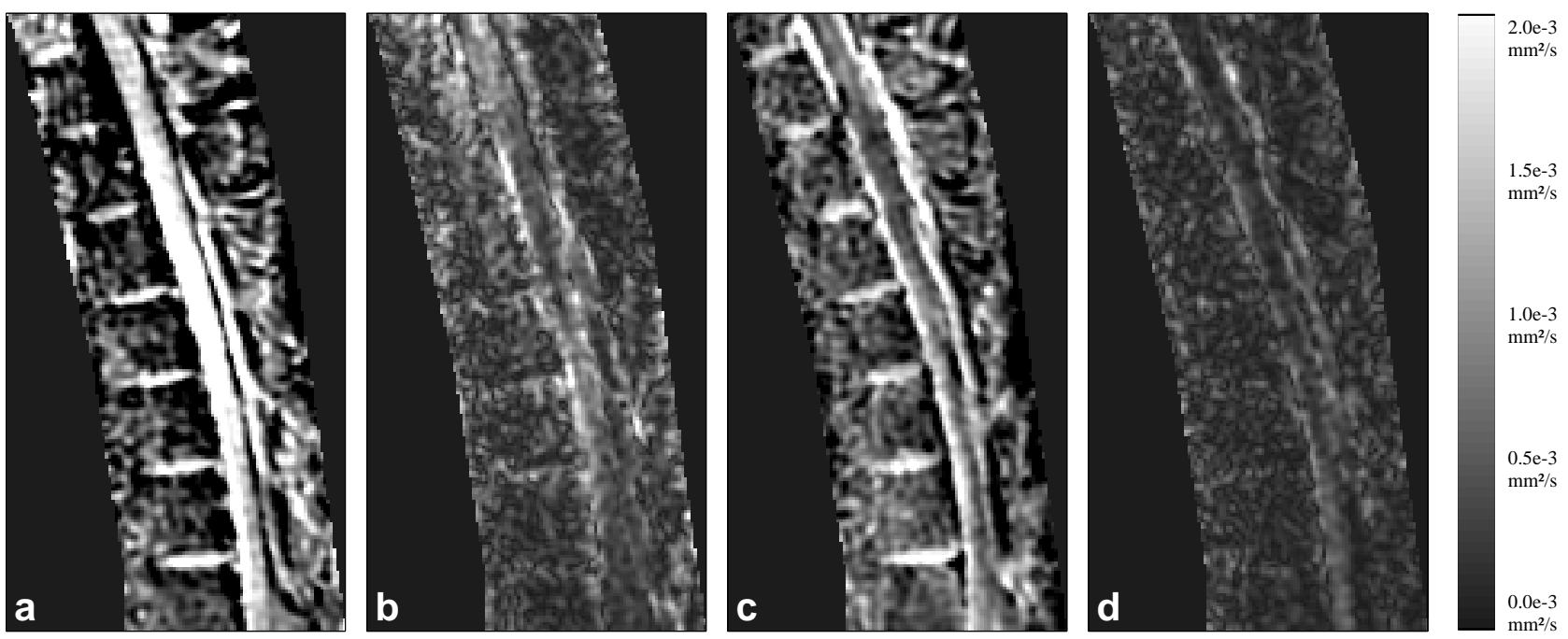

Figure 5: ADC maps of the spine with diffusion gradients in HF direction (a) and LR direction (c). The absolute error of the $A D C$ is displayed next to each ADC map (b, d). 

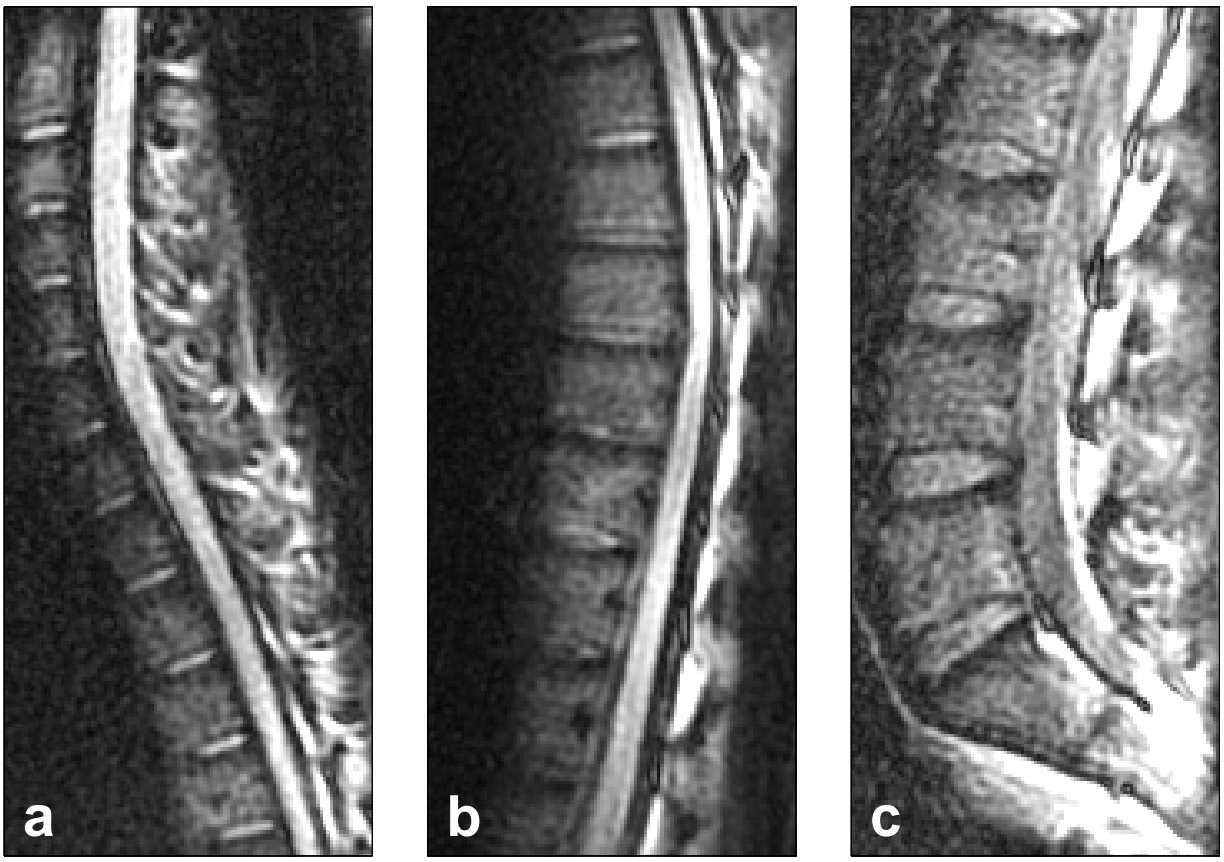

Figure 6: Diffusion-weighted images of the cervical (a), thoracic (b), and lumbar (c) spine with $b$ value $200 \mathrm{~s} / \mathrm{mm}^{2}$ in LR direction.

Alternative approaches for DWI of the cervical spinal cord have been described recently in publications by Clark et al. [36, 37] and Bammer et al. [38]. The former use conventional spin echo sequences with additional navigator echo acquisition for motion correction [18]; the latter run interleaved EPI sequences with navigator echo correction. They report mean values of the ADC in the spinal cord of $(0.55 \ldots 0.62) \cdot 10^{-3} \mathrm{~mm}^{2} / \mathrm{s}$ perpendicular to the spine and $(1.55 \ldots 2.04) \cdot 10^{-3} \mathrm{~mm}^{2} / \mathrm{s}$ in parallel direction. These values agree well with our results displayed in Table 1. ADCs of the thoracolumbar intervertebral disks have been published by Kerttula et al. [39]; their result for the ADC of the thoracic spine of $(1.4 \pm 0.3) \cdot 10^{-3} \mathrm{~mm}^{2} / \mathrm{s}$ without significant anisotropy agrees with our results as well.

We are not aware of any previously published measurements of the ADCs of the cervicothoracic intervertebral disks and the vertebral bodies. Future studies will be required in order to show whether these data may indicate pre-symptomatic abnormalities such as damage or prolapse of a disk or fractures of vertebrae. The results in [39] show both an increasing ADC and an increasing anisotropy for the disks towards the lumbar spine, perhaps indicating that these parameters increase with mechanical stress or pressure and hence maybe useful in clinical diagnosis. Our own data do not extend over a sufficiently large part of the spine to show a significant dependence of these parameters on the position.

Future development of the implemented radial spin echo sequence should extend the scheme to the acquisition of multiple spin echoes after each excitation in order to reduce the acquisition time. These echoes could either pass through the center of $k$-space as in the current implementation or could be grouped into rectangular concentric strips $[28,34]$ to obtain a larger common area for motion correction.

\section{Acknowledgments}

This work was supported in part by the German Academic Exchange Service (DAAD) and The British Council (ARC project XIII-99/38).

\section{References}

[1] Moseley ME, Cohen Y, Mintorovitch J, Chileuitt L, Shimizu H, Kucharczyk J, Wendland MF, Weinstein PR. Early detection of regional cerebral ischemia in cats: comparison of diffusion- and T2-weighted MRI and spectroscopy. Magn Reson Med 1990:14:330-346.

[2] Lövblad KO, Baird AE, Schlaug G, Benfield A, Siewert B, Voetsch B, Connor A, Burzynski C, Edelman RR, Warach S. Ischemic lesion volumes in acute stroke by diffusion-weighted magnetic resonance imaging correlate with clinical outcome. Ann Neurol 1997:42:164-170. 
[3] Schellinger PD, Jansen O, Fiebach JB, Hacke W, Sartor K. A standardized MRI stroke protocol: comparison with CT in hyperacute intracerebral hemorrhage. Stroke 1999:30:765-768.

[4] Weber J, Mattle HP, Heid O, Remonda L, Schroth G. Diffusion-weighted imaging in ischaemic stroke: a follow-up study. Neuroradiology 2000:42:184-191.

[5] Lee LJ, Kidwell CS, Alger J, Starkman S, Saver JL. Impact on stroke subtype diagnosis of early diffusion-weighted magnetic resonance imaging and magnetic resonance angiography. Stroke 2000:31:1081-1089.

[6] Kim YJ, Chang KH, Song IC, Kim HD, Seong SO, Kim YH, Han MH. Brain abscess and necrotic or cystic brain tumor: discrimination with signal intensity on diffusion-weighted MR imaging. Am J Roentgenol 1998:171:1487-1490.

[7] Sugahara T, Korogi Y, Kochi M, Ikushima I, Shigematu Y, Hirai T, Okuda T, Liang L, Ge Y, Komohara Y, Ushio Y, Takahashi M. Usefulness of diffusion-weighted MRI with echo-planar technique in the evaluation of cellularity in gliomas. $\mathrm{J}$ Magn Reson Imaging 1999:9:53-60.

[8] Tievsky AL, Ptak T, Farkas J. Investigation of apparent diffusion coefficient and diffusion tensor anisotrophy in acute and chronic multiple sclerosis lesions. Am J Neuroradiol 1999:20:14911499.

[9] Werring DJ, Clark CA, Barker GJ, Thompson AJ, Miller DH. Diffusion tensor imaging of lesions and normal-appearing white matter in multiple sclerosis. Neurology 1999:52:1626-1632.

[10] Cercignani M, Iannucci G, Rocca MA, Comi G, Horsfield MA, Filippi M. Pathologic damage in MS assessed by diffusion-weighted and magnetization transfer MRI. Neurology 2000:54:11391144.

[11] Douek P, Turner R, Pekar J, Patronas N, Le Bihan D. MR color mapping of myelin fiber orientation. J Comput Assist Tomogr 1991:15:923-929.

[12] Pierpaoli C, Jezzard P, Basser PJ, Barnett A, DiChiro G. Diffusion tensor MR imaging of the human brain. Radiology 1996:201:637-648.

[13] Jones DK, Lythgoe D, Horsfield MA, Simmons A, Williams SC, Markus HS. Characterization of white matter damage in ischemic leukoaraiosis with diffusion tensor MRI. Stroke 1999:30:393-397.
[14] Shimony JS, McKinstry RC, Akbudak E, Aronovitz JA, Snyder AZ, Lori NF, Cull TS, Conturo TE. Quantitative diffusion-tensor anisotropy brain MR imaging: normative human data and anatomic analysis. Radiology 1999:212:770-784.

[15] Chun T, Ulug AM, van Zijl PC. Single-shot diffusion-weighted trace imaging on a clinical scanner. Magn Reson Med 1998:40:622-628.

[16] Li TQ, Takahashi AM, Hindmarsh T, Moseley ME. ADC mapping by means of a singleshot spiral MRI technique with application in acute cerebral ischemia. Magn Reson Med 1999:41:143-147.

[17] Ordidge RJ, Helpern JA, Qing ZX, Knight RA, Nagesh V. Correction of motional artifacts in diffusion-weighted MR images using navigator echoes. Magn Reson Imaging 1994:12:455-460.

[18] Anderson AW, Gore JC. Analysis and correction of motion artifacts in diffusion weighted imaging. Magn Reson Med 1994:32:379-387.

[19] Latta P, Jellus V, Budinsky L, Mlynarik V, Tkac I, Luypaert R. Motion artifacts reduction in DWI using navigator echoes: a robust and simple correction scheme. MAGMA 1998:7:21-27.

[20] Dietrich O, Heiland S, Benner T, Sartor K. Reducing motion artefacts in diffusion-weighted MRI of the brain: efficacy of navigator echo correction and pulse triggering. Neuroradiology 2000:42:85-91.

[21] Clark CA, Barker GJ, Tofts PS. Improved reduction of motion artifacts in diffusion imaging using navigator echoes and velocity compensation. $\mathrm{J}$ Magn Reson 2000:142:358-363.

[22] Gudbjartsson H, Maier SE, Mulkern RV, Morocz IA, Patz S, Jolesz FA. Line scan diffusion imaging. Magn Reson Med 1996:36:509-519.

[23] Maier SE, Gudbjartsson H, Patz S, Hsu L, Lövblad KO, Edelman RR, Warach S, Jolesz FA. Line scan diffusion imaging: characterization in healthy subjects and stroke patients. Am J Roentgenol 1998:171:85-93.

[24] Gmitro AF, Alexander AL. Use of a projection reconstruction method to decrease motion sensitivity in diffusion-weighted MRI. Magn Reson Med 1993:29:835-838.

[25] Trouard TP, Theilmann RJ, Altbach MI, Gmitro AF. High-resolution diffusion imaging 
with DIFRAD-FSE (diffusion-weighted radial acquisition of data with fast spin-echo) MRI. Magn Reson Med 1999:42:11-18.

[26] Seifert MH, Jakob PM, Jellus V, Haase A, Hillenbrand C. High-resolution diffusion imaging using a radial turbo-spin-echo sequence: implementation, eddy current compensation, and selfnavigation. J Magn Reson 2000:144:243-254.

[27] Finsterbusch J, Frahm J. Diffusion-weighted single-shot line scan imaging of the human brain. Magn Reson Med 1999:42:772-778.

[28] Pipe JG. Motion correction with PROPELLER MRI: application to head motion and freebreathing cardiac imaging. Magn Reson Med 1999:42:963-969.

[29] Turner R, Le Bihan D, Maier J, Vavrek R, Hedges LK, Pekar J. Echo-planar imaging of intravoxel incoherent motion. Radiology 1990:177:407-414.

[30] Turner R, Le Bihan D, Chesnick AS. Echo-planar imaging of diffusion and perfusion. Magn Reson Med 1991:19:247-253.

[31] Trouard TP, Sabharwal Y, Altbach MI, Gmitro AF. Analysis and comparison of motioncorrection techniques in diffusion-weighted imaging. J Magn Reson Imaging 1996:6:925-935.

[32] O'Sullivan JD. A fast sinc function gridding algorithm for Fourier inversion in computer tomography. IEEE Trans Med Imaging 1985:4:200-207.

[33] Schomberg H, Timmer J. The gridding method for image reconstruction by Fourier transformation. IEEE Trans Med Imaging 1995:14:596-607.

[34] Dannels WR, Xu Y, Liu H. Rotating diffusion MR imaging reduced motion artifacts. United States Patent 1998:5,833,609.

[35] Xing D, Papadakis NG, Huang CL, Lee VM, Carpenter TA, Hall LD. Optimised diffusionweighting for measurement of apparent diffusion coefficient (ADC) in human brain. Magn Reson Imaging 1997:15:771-784.

[36] Clark CA, Barker GJ, Tofts PS. Magnetic resonance diffusion imaging of the human cervical spinal cord in vivo. Magn Reson Med 1999:41:1269-1273.

[37] Clark CA, Werring DJ, Miller DH. Diffusion imaging of the spinal cord in vivo: estimation of the principal diffusivities and application to multiple sclerosis. Magn Reson Med 2000:43:133138.
[38] Bammer R, Fazekas F, Augustin M, Simbrunner J, Strasser-Fuchs S, Seifert T, Stollberger R, Hartung HP. Diffusion-weighted MR imaging of the spinal cord. Am J Neuroradiol 2000:21:587591.

[39] Kerttula LI, Jauhiainen JP, Tervonen O, Suramo IJ, Koivula A, Oikarinen JT. Apparent diffusion coefficient in thoracolumbar intervertebral discs of healthy young volunteers. J Magn Reson Imaging 2000:12:255-260. 